\title{
Pharmacological Management of Alcohol Dependence Syndrome
}

Cite as : Doyle, L. Keogh, B. \& Lynch, A., Pharmacological Management of Alcohol Dependence Syndrome, Mental Health Practice, 14, (1), 2010, p14 - 19

Authors:

Louise Doyle RPN, BNS, RNT, MSc

Lecturer in Mental Health Nursing

School of Nursing and Midwifery

Trinity College

24 D'Olier Street

Dublin 2

Ireland

Phone: 0035318963102

Email: louise.doyle@tcd.ie

Brian Keogh RPN, BNS, MSc

Lecturer in Mental Health Nursing

School of Nursing and Midwifery

Trinity College

24 D'Olier Street

Dublin 2

Ireland

Phone: 0035318963109

Email: bjkeogh@tcd.ie

Aileen Lynch BSc, MSc, PhD

Lecturer in Biological Sciences

School of Nursing and Midwifery

Trinity College

24 D'Olier Street

Dublin 2

Ireland

Phone: 0035318968571

Email: amlynch@tcd.ie 


\begin{abstract}
The treatment of Alcohol Dependence Syndrome (ADS) generally involves strategies to manage detoxification as well as strategies to promote recovery and relapse prevention. This article describes ADS and discusses the various pharmacological interventions that can be used as part of the overall management of ADS.
\end{abstract}

Introduction

Individuals undergoing treatment for Alcohol Dependence Syndrome generally navigate their way through the phases of detoxification, recovery and relapse prevention (Moore 2008). Although detoxification is underpinned by a biological approach to treatment, psychosocial interventions such as education and peer support which often advocate abstinence are the mainstay of treatment regimes. However there are a range of pharmacological treatments available that may assist alcohol dependent individuals to successfully engage with the difficult and often lifelong processes associated with recovery and relapse prevention. The aim of this article is to discuss the drugs that can be used in the management of ADS. It will begin by introducing the concept of ADS and the physical, psychological and social consequences associated with it. The general management of ADS will also be briefly outlined. The specific drugs associated with each of the phases of ADS will be discussed with particular reference to their uses, their mode of action, side effects, interactions and any nursing implications that may be associated with their use.

Alcohol Dependence Syndrome

According to the World Health Organisation (2007:7) 'alcohol is a psychoactive substance with a known liability to cause dependence in humans and animals'. Alcohol Dependence comprises a cluster of symptoms related to the dependent individuals' inability to control their alcohol intake coupled with persistent alcohol consumption, tolerance and withdrawal symptoms if alcohol is stopped or intake reduced (APA 2000). The DSM criteria for substance dependence related to alcohol are presented below. For a diagnosis to be made, the individual should demonstrate at least three of the following symptoms in a twelve month period.

1. Tolerance: the individual drinks greater amounts of alcohol in order to achieve intoxication. In some cases tolerance may be reduced and this may reflect an inability to metabolise alcohol between consumption or underlying liver damage.

2. Withdrawal: The person may experience unpleasant symptoms if they stop drinking or if they cut down the amount they drink. These withdrawal symptoms can range from mild to life threatening.

3. The person consumes more alcohol and for longer periods than was intended.

4. The individual tries to stop or cut down drinking but is unable to do so.

5. A prolonged time is spent drinking or recovering from the effects of alcohol.

6. The person neglects important social and occupational activities or responsibilities.

7. The individual continues to consume alcohol despite an awareness of their problem and the negative physical, psychological and social impact it is having (APA 2000). 
Consequences of Alcohol Dependence Syndrome

The consequences of ADS are far reaching and can transcend the individual, his or her immediate family and their extended social network. Excessive alcohol consumption is known to damage most physiological systems most notably the cardiovascular, neurological and digestive systems. As alcohol is primarily metabolised in the liver, alcoholism can lead to liver damage, cirrhosis, eventual liver failure and death. Prolonged and heavy consumption can also result in cardiovascular problems such as cardiomyopathy and hypertension, neurological problems such as cognitive impairment and neuropathy as well as digestive problems such as stomach ulcers. Vitamin deficiencies (particularly thiamine deficiency) can cause Wernicke's encephalopathy which is characterised by confusion and short term memory problems. If left untreated, it can progress to the chronic condition of Korsakoff's syndrome (sometimes called Korsakoff's psychosis) which is characterised by memory loss and hallucinations which is often irreversible. Alcohol is also a known carcinogen and heavy drinkers put themselves at risk of developing many different forms of cancers, particularly of the mouth, oesophagus, colon and breast (WHO 2007). Psychologically, alcohol dependence is related to a range of mental health problems which are either cited as a precipitating factor to ADS or as a consequence. Decreased impulse control associated with alcohol consumption is often implicated in suicide and suicidal behaviour. At an individual social level, ADS can lead to a range of problems including loss of employment, financial problems, relationship problems, violence and aggression, neglect and social isolation. Excessive alcohol consumption is also associated with increased numbers of accidents including road traffic accidents, serious crimes and homicide.

Treatment of Alcohol Dependence Syndrome

As mentioned, treatment regimes generally follow a set pattern of detoxification, recovery and relapse prevention. The goal of most treatment programmes is either abstinence or controlled drinking, however controlled drinking may not be suitable for many people (Harniman 2006). Detoxification involves helping the individual to physically withdraw from alcohol safely. Depending on the individual's alcohol consumption, their physical condition and their previous experience of detoxification, this can be done either in hospital or at home. Withdrawal symptoms include tremor, sweats, anxiety, gastrointestinal upsets and insomnia. More serious symptoms such as seizures and delirium tremens necessitate hospitalisation (Harniman 2006). Seizures can affect up to one third of individuals withdrawing from alcohol and for most they resolve without further complications. However some people may develop status epilepticus which is life threatening (Rogawski 2005). Delirium tremens, more commonly known as the DTs, consists of clouded consciousness, poor concentration, confusion and disorientation (APA 2000). It has a rapid onset and there is often fluctuation between levels of consciousness with periods of lucidity being peppered with periods of confusion and marked agitation. It is difficult to predict which individuals will develop delirium tremens or seizures; a prior history of either during detoxification is the strongest indicator of them occurring again.

Psychosocial interventions dominate the recovery and relapse prevention phases of treatment and consist of strategies such as education programmes, cognitive 
behaviour therapy and motivational interviewing. Key to these interventions is the desire to get to the root cause of the problematic drinking behaviour. Twelve step programmes such as Alcoholics Anonymous are renowned throughout the world and advocate abstinence through a peer support programme. Other interventions such as social skills and vocational training may also be helpful for individuals with ADS who have been out of employment or who are in need of retraining.

Pharmacological treatment of Alcohol Dependency Syndrome

Medical and nursing interventions, in particular pharmacological treatment, have a significant role to play in the management of ADS. There has been an increased interest in pharmacological treatment options for a variety of reasons including that significant advances have been made in our understanding of the way substances act on the brain and the chemical changes that can underlie psychological processes (Merrill 2002). This in turn has resulted in an increased concentration on the field of pharmacology of substance use which has resulted in the development of new drug therapies. Traditionally, the use of medications in the treatment of alcohol dependency syndrome was mainly limited to the detoxification stage, with little or no pharmacological intervention occurring in the relapse prevention and recovery phases of treatment programmes. These advances in pharmacotherapy however have resulted in the use of pharmacological interventions for all stages of ADS. Nevertheless, it is important to remember that pharmacological treatments are only one aspect of the full treatment regime for $\mathrm{ADS}$ and that other interventions including nursing care are essential to the success of a treatment programme. McBride (2002) points out that none of the adjunctive drug treatments have been shown to work effectively in the absence of psychosocial intervention.

\section{Pharmacological treatment of withdrawal and detoxification in ADS}

The initial use of medication in ADS is concerned with getting the client through the often very difficult withdrawal and detoxification phase of treatment. As previously identified, alcohol withdrawal syndrome presents differently for different people and depends to a large degree on how much and how long a person has been drinking prior to the cessation of drinking. Withdrawal symptoms will usually occur within a matter of hours of abstinence and will usually peak within the first 72 hours. During this period, the first drugs often prescribed to a person withdrawing from alcohol are long-acting Benzodiazepines. Benzodiazepines are one of the most commonly prescribed psychotropic drugs due mainly to the fact that they have many indications for use. They are primarily used as an anxiolytic but have many other uses including as a muscle relaxant and as a sedative. They are used in ADS to attenuate withdrawal symptoms and allow for the abrupt cessation of alcohol use. The neurotransmitter gamma-amino butyric acid (GABA) acts via the GABA receptor complex and exerts an overall inhibitory effect on the brain. The benzodiazepines act by binding to benzodiazepine receptors which comprise part of the larger GABA receptor complex. Because alcohol decreases GABAergic neurotransmission the mode of action of benzodiazepines is to potentiate the inhibitory action of GABA in the brain. The two most commonly used Benzodiazepines in ADS are Chlordiazepoxide (Librium) and Diazepam (Valium). There are a number of concerns about using benzodiazepine medication in the treatment of ADS but the most pressing one is the fact that these drugs are themselves highly addictive and therefore the risk of cross-addiction between alcohol and benzodiazepines is high. For this reason, administration of benzodiazepines should be for a limited period only. Furthermore, benzodiazepines 
should not be prescribed if the client is likely to continue drinking alcohol as there is the risk of respiratory depression with continued alcohol and benzodiazepine use. There are two main dosing regimes when using benzodiazepines for alcohol withdrawal and these are (i) the sliding scale and (ii) symptom triggered therapy. Using the sliding scale regime, a relatively high dose is given on day one and is subsequently reduced over 7-14 days depending on the severity and persistence of the withdrawal symptoms e.g chlordiazepoxide 10-50 mgs 4 times daily (BNF 2008) or Diazepam 40-80 mgs per day in four divided doses (McBride 2002). McBride (2002) points out that there is some flexibility with this regime in that the dose can be increased if the client demonstrates severe withdrawal symptoms or it can be decreased if over-sedation is apparent. However, the risk of over-dosing or underdosing the client is still there and this had led to the use of symptom triggered therapy. Using this regime the medication is given every 90 minutes (e.g. Diazepam 20 mgs every 90 minutes) for as long as the person experiences significant withdrawal symptoms as rated by a trained observer. McBride (2002) suggests the use of a recognised withdrawal scale to determine the severity of withdrawal symptoms over time and identifies that by using this system the average client receives only around $80 \mathrm{mgs}$ of Diazepam. The benefit of using the symptom triggered therapy method of dosing is that it responds to each client's individual needs in relation to withdrawal rather than applying a blanket dosing regime regardless of need.

Withdrawal seizures may be a feature of a severe alcohol withdrawal syndrome. If this is the case the use of Diazepam can help to alleviate this symptom and can be given rectally if required, therefore avoiding the risk of polypharmacy. However, McBride (2002) identifies that people with a history of withdrawal fits may be prescribed an anti-convulsant in addition to the drug prescribed for withdrawal. In this case, Carbamazepine (Tegretol) or Sodium Valporate (Epilim) are the drugs of choice. Carbamazepine promotes sodium efflux from nerve cells which reduces the possibility that an impulse will be generated and hence slows neurotransmission. Sodium valporate promotes the release of GABA at the synapse so more GABA binds to the GABA receptor complex and increases GABA neurotransmission. Therefore both drugs act on different targets to exert an overall inhibitory effect on the brain. Status epilepticus is a medical emergency and urgent treatment is required. Intravenous Lorazepam or Diazepam is usually prescribed under strict medical and nursing supervision. In some cases Diazepam given rectally may also be prescribed.

Another group of drugs often commenced during the withdrawal/detoxification stage of treatment are vitamins. There are often long-standing nutritional deficiencies in people who are alcohol dependent. This happens for a number of reasons including that many individuals with a drinking problem have limited food intake, alcohol reduces the absorption of many of the vitamins and also getting a nutritionally sound diet is often not a priority for a person who drinks heavily. As previously identified, vitamin deficiencies (particularly vitamin B1 - thiamine) can result in serious neurological problems such as Wernicke's encephalopathy and Korsakoff's syndrome. As a result, it is important that any vitamin deficiencies are addressed in the early stages of treatment for ADS. Initial vitamin supplementation may take the form of parenteral treatment used for rapid correction of severe depletion or malabsorption of vitamins. In these cases, Pabrinex given intramuscularly is most often used. It is important to note that when administering parenteral thiamine such as Pabrinex, nurses and other health care staff should be vigilant for signs of anaphylaxis 
and resuscitation equipment should be available on administration (BNF 2008). Following this initial treatment, thiamine or other oral vitamin preparations may be used in the longer term (BNF 2008). In general, vitamins act as coenzymes which are necessary for optimal enzymatic activity. Therefore vitamins, particularly thiamine, are essential for the complete aerobic release of energy from carbohydrates and this energy is needed to fuel all the cells of the body, especially neural cells. A deficiency of thiamine leads to incomplete metabolism of carbohydrate and results in the accumulation of lactic and pyruvic acids, which in turn can cause oedema, heart failure and the neurological symptoms mentioned above. An important nursing consideration to bear in mind here is that many clients may overlook the importance of taking their vitamin medication believing them not to be important. Therefore client education about the role of these vitamins in the prevention of neurological damage is imperative here.

Pharmacological treatment of relapse prevention and management of ADS

Once the alcohol has been cleared from the system and most of the physical effects of withdrawal have been combated, it is vital to consider the very important issue of deterring relapse and maintaining abstinence. The metabolism of alcohol requires the sequential action of two liver enzymes, alcohol dehydrogenase and aldehyde dehydrogenase to convert it to acetic acid and these enzymes are illustrated in Figure 1 .

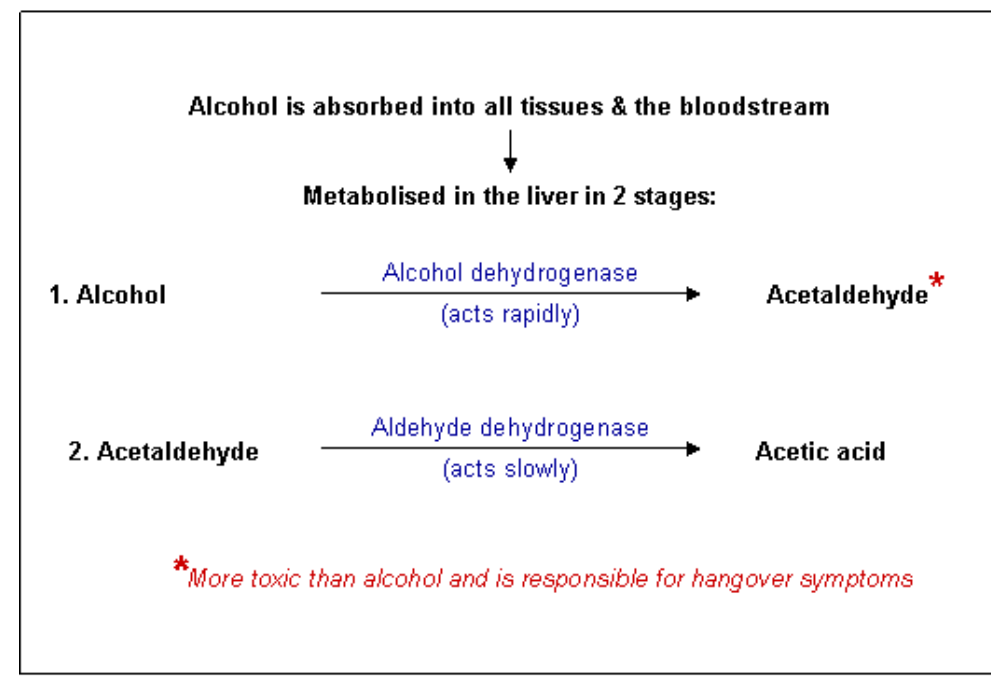

Figure 1: Metabolism of alcohol

One drug used to deter relapse is Disulfiram (Antabuse). When alcohol is consumed, Disulfiram inhibits the action of aldehyde dehydrogenase (Figure 1, step 2) causing a rise in the blood levels of acetaldehyde known as the Disulfiram-Ethanol Reaction. The accumulation of acetaldehyde (a noxious substance) is responsible for many adverse symptoms and includes:

- Violent throbbing headache

- Nausea and vomiting

- Chest pain

- Palpitations

- Breathlessness

- Flushed face

- Tachycardia 
- Arrthymias

- Decrease in blood pressure leading to vertigo, blurred vision and possible eventual collapse in severe cases.

This reaction can occur within 10 minutes of consuming alcohol and can last several hours. Very small amounts of alcohol can induce a reaction so the nurse should educate the client about taking medications that contain alcohol such as cough mixtures, the use of certain toiletries such as mouthwashes and the presence of alcohol in some foods. Furthermore, it is imperative that alcohol is not consumed for at least 24 hours prior to commencing treatment with Disulfiram and should be avoided for at least one week after stopping treatment (BNF 2008). Clients should also be warned about the dangers associated with drinking through the acetaldehyde reaction. The common side-effects of Disulfiram include initial drowsiness and fatigue, nausea and vomiting and halitosis. McBride (2002) contends that Disulfiram is only of potential value in those whose intention it is to abstain from alcohol but who lapse frequently and therefore become disheartened and frustrated. In this instance, Disulfiram acts as a form of an external motivating factor to avoiding alcohol that day, as the knowledge is there that if they drink alcohol they will experience this unpleasant reaction. Treatment with Disulfiram works best when the drug is administered under supervision. This drug should not be given to those who have no real intention or desire to abstain from alcohol. Using Disulfiram gives the client a period of stability in which they are alcohol free enabling them to sort out some of the problems that ADS presents for them. In addition it allows them the opportunity to consider the options about their future and to engage with psychosocial interventions to remain alcohol free if that is the desired goal.

While Disulfiram as a pharmacological agent can help the person to become alcohol free, other drugs are used to help the person remain off alcohol. Acamprosate (Campral) is one such drug and it's main indication for use is the reduction of craving for alcohol and therefore the maintenance of abstinence. Glutamate is the main excitatory neurotransmitter and alcohol increases glutamatergic activity by increasing the density of NMDA glutamatergic receptors in the brain. When alcohol is withdrawn and during early abstinence, there is a compensatory surge in glutamate release at the synapse and Acamprosate acts by decreasing this glutamatergic surge and normalises the glutamatergic excitation. This effect may reduce craving and distress and may thus decrease the need to consume alcohol (Swift 1999). The client should be alcohol free before treatment is commenced and treatment is recommended for six months to one year.

Another drug used in the treatment of ADS is Naltrexone. Naltrexone is an opioid antagonist. Opioid antagonists work by blocking the effects of the body's own natural opioid-like substances, the endorphins, as well as the effects of other opioid drugs (McBride 2002). One of the effects of alcohol use is to release endorphins into the brain hence providing the pleasant sensations associated with alcohol use. If Naltrexone is consumed before every drinking session then this will inhibit the production of endorphins so that the person does not experience the pleasant feelings normally associated with their alcohol use. In other words clients who are alcohol dependent have less alcohol highs when drinking while taking Naltrexone. Therefore, after a period of time the association between alcohol and pleasure is broken and drinking alcohol will be reduced. An important nursing consideration here is that 
clients should be made aware that in the event of severe pain, the effect of other opioid analgesics will be blocked if the client has taken Naltrexone (McBride 2002). Therefore, if admitted to hospital following an accident or trauma other opioid analgesics will not be effective in alleviating pain and the treating team should be made aware of this.

\section{Conclusion}

The development of an international action plan targeting the reduction of the harmful effects of alcohol consumption is a key recommendation of the World Health Organisation (2007). Although national and international policies advocate a more responsible approach to alcohol consumption, it is likely that ADS will continue to be a significant health problem. Pharmacological approaches as discussed in this article have an important role to play in the overall management of ADS. However they must be used in conjunction with psychosocial intervention to maximise successful outcomes.

\section{References}

American Psychiatric Association (2000) DSM-IV-TR: Diagnostic and Statistical Manual of Mental Disorders. American Psychiatric Association Washington.

British Medical Formulary and Royal Pharmaceutical Society of Great Britain (2008) British National Formulary. British Medical Formulary and Royal Pharmaceutical Society of Great Britain, London

Harniman B (2006) Substance misuse: an overview of assessment and treatment options. Nurse Prescribing. 2, 5, $180-183$.

McBride, A. (2002) Medical Approaches and Prescribing: Alcohol. in Petersen T. \& McBride, A. (Eds) (2002) Working with Substance Misuers: A Guide to Theory and Practice. Routledge, London.

Merrill, J. (2002) Medical Approaches and Prescribing: Drugs. in Petersen T. \& McBride, A. (Eds) (2002) Working with Substance Misuers: A Guide to Theory and Practice. Routledge, London.

Moore G (2008) Working with addiction problems in Morrissey J, Keogh B, Doyle L (Eds) (2008) Psychiatric Mental Health Nursing: An Irish Perspective. Gill \& Macmillan, Dublin.

Rogawski M (2005) Update on the neurobiology of alcohol withdrawal seizures. Epilepsy Currents. 5, 6, 225 - 230.

Swift, R.M. (1999) Drug Therapy for Alcohol Dependence. The New England Journal of Medicine 340(19), 1482-1490.

World Health Organisation (2007) WHO expert committee on problems related to alcohol consumption, second report. WHO, Geneva. 